\title{
Estrategia pedagógica mediada por TIC para el aprendizaje de la mecánica respiratoria, considerando situaciones problémicas y estilos de aprendizaje
}

\section{ICT mediated pedagogic strategy to learn respiratory mechanics taking account of problem-solving situations and learning styles}

\author{
Ruby Jackeline Rey Triana \\ Enfermera. Especialista en Epidemiología \\ Magíster en Educación (c). \\ Docente de la Universidad Autónoma de Bucaramanga \\ Santander, Colombia \\ Correo electrónico: rubjackt@gmail.com \\ Hernán Darío Delgado Rico \\ Médico. Magíster en Educación \\ Docente Asociado Universidad Autónoma de Bucaramanga \\ Magíster en Ciencias Biomédicas por la Universidad Industrial de Santander, Colombia \\ Correo electrónico: hdelgado3@unab.edu.co \\ Erika Yurley Durán Niño \\ Enfermera Universidad de Santander \\ Magíster en Educación (c). Universidad Autónoma de Bucaramanga, Colombia \\ Docente Universidad de Santander, Colombia \\ Correo electrónico: erikayurley17@hotmail.com
}

Recibido 3 de agosto de 2016; aprobado 30 de agosto de 2016

\section{Cómo citar este artículo:}

Rey, R.J., Delgado, H.D., y Durán, E.Y. (2017). Estrategia pedagógica mediada por TIC para el aprendizaje de la mecánica respiratoria, considerando situaciones problémicas y estilos de aprendizaje. Espiral, Revista de Docencia e Investigación, $7(1), 11-26$

\section{Resumen}

Los avances en el conocimiento y desarrollos tecnológicos actuales exigen cambios en la Educación, reorientándola hacia un papel protagónico del estudiante en el aprendizaje autónomo, significativo y colaborativo junto a docentes reflexivos y abiertos a la búsqueda de nuevas estrategias. La educación médica comparte estos retos que demandan médicos críticos, adaptativos y que entiendan y utilicen recursos tecnológicos para resolver problemas de salud en sus pacientes y poblaciones.

Objetivo. Diseñar una estrategia pedagógica basada en situaciones problémicas y estilos de aprendizaje, mediada por TIC, para mejorar el desarrollo de competencias sobre la Mecánica Respiratoria.

Metodología. Diseño con un grupo experimental y uno de control, con mediciones antes y después en ambos grupos. Población: estudiantes de tercer semestre de Medicina en una universidad colombiana.

Resultados. El grupo que tomó la estrategia basada en situaciones problémicas con materiales adaptados al estilo de aprendizaje y mediada por TIC, mejoraron en comparación con el grupo de control $(p<0.001)$; hubo mejor rendimiento global en el grupo de intervención ( $p$ $=0.017$ ). El uso de la estrategia pedagógica no mejoró la memorización de contenidos comparándose con el control $(p=0.145)$. La satisfacción de la estrategia se encontró en alto grado.

Conclusiones. Esta estrategia es una alternativa novedosa e innovadora, incorpora las TIC para el aprendizaje individual y colectivo en mecánica respiratoria. Debe existir diversidad en las estrategias de evaluación que no se limiten a valoraciones sumativas de tipo cuestionario que desconocen otros procesos de evaluación relacionados con aprendizaje significativo.

Palabras claves: Educación superior, estilos de aprendizaje, tecnologías de información y comunicación, mecánica respiratoria, situaciones problema. 


\begin{abstract}
Advances in knowledge and current technological developments require changes in education, redirecting it towards a student's leading role in autonomous, meaningful and collaborative learning with thoughtful teachers and open to the search for new strategies. Medical education shares these challenges that demand critical, adaptive physicians who understand and use technological resources to solve health problems in their patients and populations.
\end{abstract}

Objective. Was To design a pedagogical strategy based on problems situations and learning styles, mediated by ICT, to improve the development of competencies on respiratory mechanics.

Methodology. It was designed with an experimental group and a control one, with measurements before and after in both groups. Population: Third semester medical students at a Colombian university.

Results. The group that took the strategy based on problematic situations with materials adapted to the learning style and mediated by ICT, improved in comparison with the control group $(p<0.001)$; There was a better performance in the intervention Group $(P=0.017)$. The use of the pedagogical strategy did not improve the memorization of contents compared with the control $(P=0145)$. The satisfaction of the strategy was found in high degree.

Conclusions. This strategy is a new and innovative alternative, incorporating ICT for individual and collective learning in respiratory mechanics. There should be diversity in evaluation strategies that are not limited to questionnaire-type summative assessments that do not know other evaluation processes related to meaningful learning.

Keywords: Higher education, learning styles, information and communication technology, respiratory mechanics, problem solving.

\section{Introducción}

En una sociedad altamente competitiva, acelerada y globalizada, juegan un papel importante tanto las políticas gubernamentales en materia educativa, como el compromiso de las instituciones por garantizar y motivar el aprendizaje de los estudiantes. Los enfoques educativos en la actualidad propenden dejar de enfatizar en estrategias centradas en el docente, propias de la educación tradicional, orientadas a inducir el aprendizaje de contenidos (interpretar, analizar y memorizar), para fomentar más la participación del estudiante en su aprendizaje, involucrándolo directamente en actividades que le permitan desarrollar el pensamiento crítico y en consecuencia, autorregular su propio aprendizaje.

A lo largo de la historia diversos paradigmas han influenciado la educación, cada uno de ellos soportado en la psicología, filosofía y sociología, pero todos con el único fin de desarrollar en el estudiante la inteligencia, las capacidades, las destrezas, el aprendizaje y los valores. Dentro de los paradigmas que han influenciado la educación conviene mencionar: el conductual, basado en la conducta-comportamiento; el paradigma cognitivo, que ofrece al estudiante las herramientas para trabajar activamente en la construcción del conocimiento y el docente actúa como facilitador, y el paradigma de competencias, en el que el estudiante además de participar activamente en la adquisición del conocimiento, cuenta con un ingrediente adicional que es la tecnología, la cual facilita al estudiante además de procesar, interiorizar y convertir la información en conocimiento, estar actualizado permanentemente para poder enfrentar los retos del ejercicio profesional (Duque \& Perdomo, 2014).

Como resultado de la globalización y sus efectos sociales, se hace necesario dar un cambio de paradigma impulsado por la sociedad del conocimiento, para fundamentar los procesos de enseñanza y aprendizaje en el rol activo del estudiante, que permita desarrollar sus competencias de manera autónoma y vivencial con estrategias guiadas por el docente, dando importancia a la enseñanza por competencias enfocadas en el saber ser y saber estar (genéricas) y en el saber y saber hacer (específicas). Por ello, la educación superior europea al adoptar el aprendizaje por competencias, menciona que las universidades deben proponer métodos de innovación pedagógica para lograrlo (Díaz, 2006)

Para Byrne \& Rozental (1994) las escuelas médicas no deben estar lejanas a estos para- 
digmas educacionales, pues su gran objetivo es formar profesionales que aporten beneficios a la sociedad, que puedan integrarse a los nuevos cambios y retos que traen los avances científicos y tecnológicos, ofreciendo una atención con calidad, es por ello que los procesos de enseñanza deben estar integrados permitiendo un aprendizaje basado en la realidad, en las vivencias propias, colaborativas y prácticas.

Motivar la vinculación "natural" de un estudiante a una actividad de aprendizaje que implique a su vez el desarrollo de una competencia y el logro de aprendizajes significativos, exige la consideración de aspectos como el estilo de aprendizaje del estudiante (Felder \& Spurlin, 2005), en el diseño u organización de los contenidos de apoyo y la valoración del nivel de conocimiento, para medir el avance del estudiante en su aprendizaje dentro de las actividades de formación previstas, así como el contexto y los presaberes de los estudiantes.

Este trabajo propone una estrategia pedagógica basada en situaciones problémicas para orientar el aprendizaje mediado por TIC, de la Mecánica Respiratoria (un módulo del curso Fisiología Humana, del programa de Medicina de una universidad colombiana), considerando como variables el estilo de aprendizaje y el nivel de conocimiento de los estudiantes. El estilo de aprendizaje detectado en el cuestionario desarrollado por Felder y Soloman es importante para el diseño y construcción de los contenidos de apoyo que ofrece el docente, y el nivel de conocimiento para "graduar" el avance del estudiante a través del currículo del curso (Felder \& Soloman, 2005; Felder \& Spurlin, 2005)

Para la realización de la estrategia se partió de tres corrientes contemporáneas: a) el Constructivismo en el que está presente la pedagogía de la autonomía, que resalta el papel del estudiante como protagonista de su propio aprendizaje, el cual se logra mediante la aplicación de diversas estrategias y el acompaña- miento de los docentes. El objetivo fundamental de este tipo de aprendizaje es que el estudiante aprenda de forma independiente y estratégica (Crispin et al., 2011); b) las pedagogías críticas que propenden por una educación libre y activa desde todos sus contextos: individual, social, económico y político. Para Freire (2004) la educación es influenciada por el mundo y el contexto, donde el estudiante asume el rol de actor activo con diferentes capacidades en las que es capaz de desenvolverse, haciendo uso de su historia, cultura y sociedad. Resalta que la educación es problematizadora, crítica y libertadora; c) el Conectivismo, definido como la teoría del aprendizaje en la era digital donde el caos (comprendido como toda la información que se encuentra inmersa en la red) necesita de nuevas formas de aprender, afines a los mecanismos cerebrales del estudiante y a los mecanismos propios de la red, estableciendo conexiones y patrones entre los diferentes nodos informativos (Siemens, 2014).

De la necesidad de implementar de forma correcta la tecnología surge el modelo conocido con el nombre de b-learning. Este tiene sus orígenes en las teorías del conductismo, constructivismo, cognitivismo y humanismo; en las que se busca que el estudiante indague, explore y construya conocimiento a partir de diferentes herramientas, desarrollando un aprendizaje significativo que integre diferentes prácticas pedagógicas (Vera, 2008). Implementar la tecnología junto con los procesos educativos, tal como lo propone la educación b-learning, requiere de una planeación que le permita al docente integrar de forma eficaz la tecnología en las clases dentro del aula. Para que este proceso sea posible se hace necesaria la combinación de tres componentes o dimensiones esenciales del conocimiento básico: contenido (content knowledge - CK), pedagogía (pedagogical knowledge - PK) y tecnología (technological knowledge - TK). De esta interacción resulta el modelo llamado Technological Pedagogical Content Knowledge (TPACK). La interacción de 
estos componentes permite que cada disciplina sea enseñada de una manera eficaz, con herramientas tecnológicas apropiadas, todo esto soportado en la pedagogía (Koehler, Mishra, Akcaoglu \& Rosenberg, 2008).

\section{Metodología}

El tipo de investigación es un diseño con un grupo experimental, un grupo de control y mediciones antes y después en ambos grupos, para finalizar se hacen las comparaciones entre los dos grupos de las medidas pre y pos (Briones, 1996).

El estudio se desarrolló en tres fases: la primera, el diseño de la estrategia teniendo en cuenta el modelo TPACK; segunda, la implementación dentro de la unidad sobre mecánica respiratoria, con asesoría del docente y dirigida a estudiantes de Ciencias de la Salud a nivel universitario; y tercera, la evaluación del rendimiento académico de los estudiantes frente a la estrategia utilizada, comparándolo con el grupo control que recibió la clase magistral.

El presente estudio tuvo un componente de evaluación cuantitativa en el cual los grupos de participantes $(n=80)$ estuvieron conformados por 52 mujeres (65\%) y 28 varones (35\%), quienes se distribuyeron equitativamente en 40 integrantes para cada grupo; de todos se obtuvo el consentimiento informado para participar. Estos fueron elegibles, considerando como criterios de exclusión la cancelación de semestre según los términos de la institución, estudiantes que estén repitiendo el curso y el deseo de no participar. El promedio de edades fue de 19 años, siendo 16 años el valor mínimo y 22 el valor máximo.

Al inicio del semestre se aplicó a todo el grupo el Instrumento de diagnóstico de los estilos de aprendizaje según el modelo FSLSM (Index of Learning Styles, ILS), diseñado por Richard Felder y que consiste en una batería de 44 preguntas con doble opción de respuesta, que permite evaluar las 4 dimensiones propuestas por el FSLSM, junto con las categorías.

A continuación se realizó un muestreo por conglomerados debido a que la población de estudiantes se encontró distribuida en secciones de 20 a 22 estudiantes, por lo cual se tomaron diversos estudiantes de cada uno, teniendo en cuenta que esta fuera homogénea y se organizaron en un grupo control $(n=40)$ y uno de intervención ( $n=40)$; no se tuvo en cuenta el resultado del ILS para asignar a los estudiantes a los grupos de intervención o control. Quienes fueron excluidos del grupo de intervención, eran ubicados en el grupo de control.

Los estudiantes del grupo de control recibieron la metodología utilizada tradicionalmente en la Facultad de Medicina, que consiste en una clase magistral en un auditorio y esta actividad es previa a los talleres prácticos y de resolución de problemas. Por otra parte, en el grupo de intervención, se utilizó una estrategia pedagógica (usando la plataforma Moodle) durante el desarrollo de la clase y del tema del sistema respiratorio, este grupo pudo ver los contenidos de la clase en un formato especialmente diseñado, teniendo en cuenta sus estilos de aprendizaje, así como resolver actividades y situaciones problemas que le permitieran adquirir un pensamiento crítico al momento de escoger un diagnóstico médico. El estudiante tuvo la oportunidad de revisar la plataforma de manera autónoma dentro de la institución y en el tiempo de trabajo independiente, las veces que lo consideró necesario; asimismo, tuvo la oportunidad de hacer a los docentes las preguntas que consideraron necesarias, porque en la administración de la estrategia pedagógica se contó con la presencia de uno de los docentes en un tiempo y espacio predeterminados.

El test de conocimientos fue usado en dos ocasiones: pre-test (prueba de presaberes) y pos-test (al finalizar el módulo), los cuales fueron aplicados de forma individual a cada 


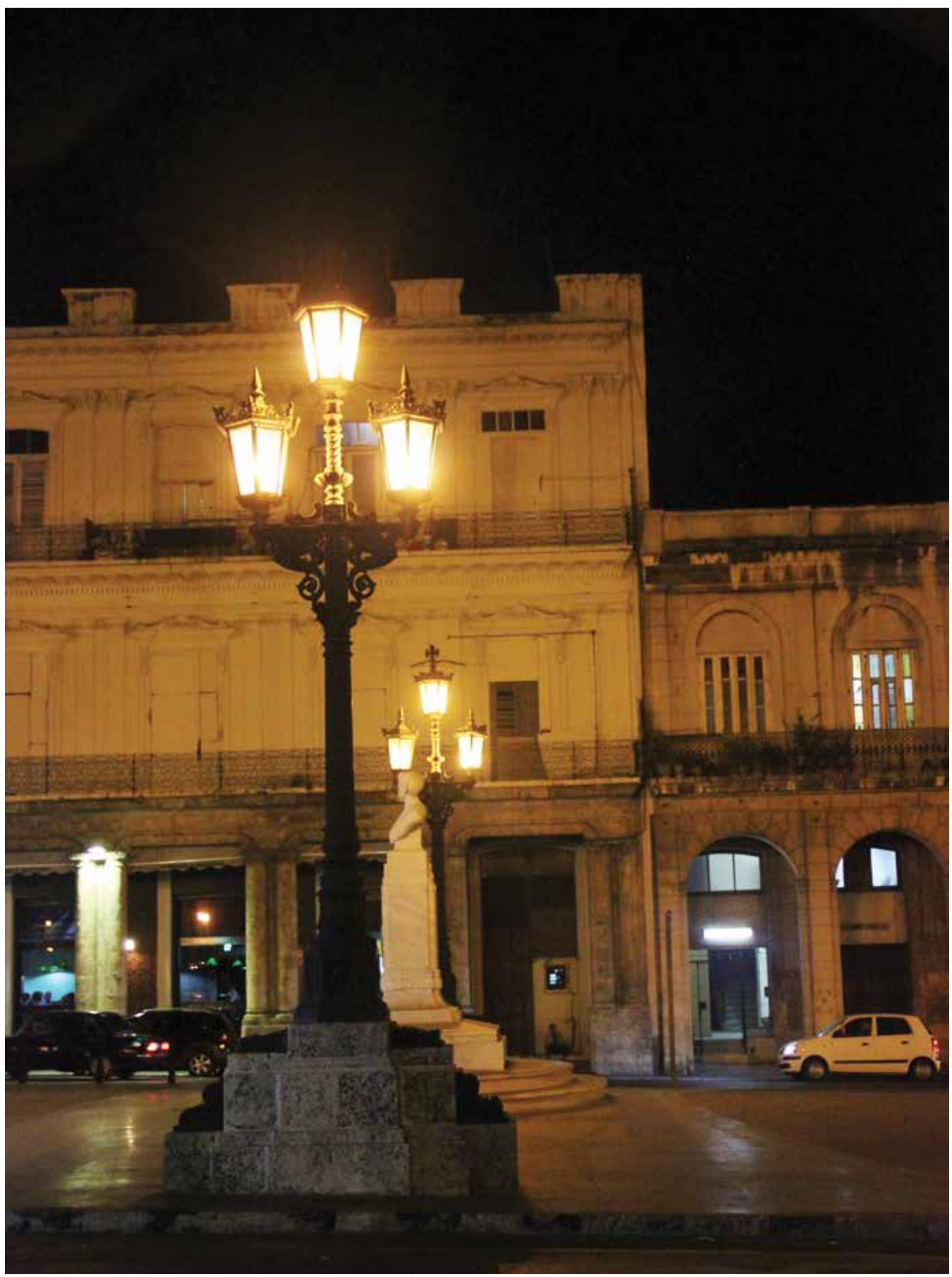


estudiante y se permitió que pudieran expresar libremente sus ideas, así como se aplicó de forma colectiva un test en la resolución de las preguntas problemas.

El procedimiento de la investigación realizada se lista en pasos sucesivos:

1. Diseño de la estrategia pedagógica: revisión de la literatura, del currículo, diseño del micro currículo a través del análisis funcional (DOA, estructuración modular, competencias, planeación curricular, guías de medios didácticos y rúbricas de evaluación), recopilación, adaptación y construcción del material puesto en la plataforma Moodle.

2. Implementación de los materiales (lecturas, material interactivo, esquemas mentales, situaciones problémicas, herramientas interactivas, entre otros) en la plataforma Moodle.

3. Obtención de consentimientos informados.

4. Aplicación de cuestionario libre de los estilos de aprendizaje (ILS) según Felder y Silverman para los estudiantes asignados al grupo de intervención y de control. Se hizo el análisis de la información en una hoja de cálculo electrónica, clasificando a cada estudiante según su estilo de aprendizaje predominante.

5. Realización del pre-test o prueba escrita de conocimientos a los dos grupos: intervención y control.

6. Liberación del material de la estrategia pedagógica para el aprendizaje de mecánica respiratoria a quienes fueron asignados a este grupo y de forma concomitante se dio la clase magistral al grupo de control; el acceso a los materiales de Moodle se permitió ad libitum, pero con- trolando el acceso de los usuarios como primera entrada al material asignado según el estilo de aprendizaje predominante que el estudiante arrojó en el ILS. El material se revisó en el aula de clase y fuera de esta, en el tiempo de trabajo independiente.

7. Actividad práctica de resolución de situaciones problémicas. Esta actividad se realizó de forma grupal.

8. Prueba escrita de conocimientos a los cinco días después de haber tomado la instrucción, se administró en un mismo momento y lugar a todo el grupo de estudiantes, independiente de la modalidad de instrucción asignada.

9. Recopilación y análisis de resultados.

Los desenlaces por considerar fueron relacionados con la Evaluación del aprendizaje, verificado por los resultados obtenidos en una prueba que involucró preguntas de selección múltiple y resolución de situaciones problemas por parte de los estudiantes, con el propósito de encontrar aprendizajes significativos. Para cuantificar los resultados se utilizó el valor predeterminado de 3.0 (tres punto cero) sobre un total de 5.0 como indicador de aprobación de la prueba. Un 35\% fue asignado a la actividad de resolución de situaciones problémicas, donde se evaluó tanto el desempeño individual como grupal, y un $65 \%$ al test de conocimientos final. Este cuestionario se administró a todos los estudiantes participantes en la investigación a los cinco días después de haber tenido su clase en el salón o bien haber utilizado los materiales de la estrategia pedagógica adaptativa; el pre-test consta de 5 preguntas y se brindó un tiempo de 10 minutos para responderlo. El pos-test se realizó a las dos semanas de hecha la intervención, constó de 5 preguntas estrechamente relacionadas con el pre-test, y se dio un tiempo de 2 minutos por pregunta. 


\section{Análisis de datos}

Los participantes fueron comparados en virtud del resultado de una prueba de conocimientos sobre los temas vistos en la clase, así hubiese participado en el grupo de intervención o en el grupo de control. La información fue recolectada a través de los instrumentos de evaluación que se desarrollaron durante la primera fase del estudio. Los resultados de su aplicación se ingresaron de manera independiente con doble digitación en una hoja de cálculo electrónica. Se hizo la depuración de datos y consolidación de la base de datos por analizar.

Los datos se presentaron con medidas de tendencia central para las variables numéricas acuerdo a la curva de normalidad se utilizaron para distribuciones de tipo normal: medias y sus desviaciones estándar; para distribuciones de tipo no normal: medianas y rangos. Para el caso de las variables categóricas medidas de frecuencia y porcentajes. La curva de normalidad se realizó con la prueba Shapiro-Wilk. Se llevó a cabo un análisis de asociación de variables con los métodos estadísticos apropiados según su naturaleza y distribución. Se exploró la relación de dependencia entre las variables dependientes (rendimiento: entendido como mejora de competencias de forma individual y grupal) y la independiente (estrategia pedagógica) en busca de asociaciones. Se consideró un valor de $\mathrm{p}$ menor a 0.05 como significativo.

En lo concerniente con las hipótesis del presente estudio experimental, se consideró desde varias perspectivas la mejora del aprendizaje de la mecánica respiratoria en los estudiantes de medicina:

- En primera instancia, se valoró como variable dependiente la diferencia entre el puntaje obtenido por estudiante en el posttest y el puntaje obtenido en el pre-test (evaluación de los presaberes), teniendo $H O=$ no existe una diferencia estadísticamente significativa en el conocimiento en- tre el grupo que tomó instrucción usando la estrategia respecto al grupo de control.

- Segundo, al realizarse dentro del módulo una actividad de resolución de problemas por equipos (ABP), se evaluó el desempeño de los grupos que se conformaron para dicha actividad teniendo presente que se agruparon siempre a los estudiantes según habían recibido la intervención o si pertenecían al grupo de control y nunca se hicieron grupos combinados, siendo así la $\mathrm{HO}=$ el uso de la estrategia basada en situación problémica y TIC no mejora el aprendizaje del estudiante para la resolución de problemas de la mecánica respiratoria.

- Finalmente, la calificación final del módulo de mecánica respiratoria contempló por diseño curricular del curso ofrecido a los estudiantes, una ponderación de $65 \%$ al resultado de su desempeño individual (prueba post) y $35 \%$ al desempeño grupal (ABP) quedando así la $\mathrm{HO}=$ el uso de la estrategia basada en situación problémica y TIC no mejora el rendimiento global del estudiante en mecánica respiratoria.

Se consideró un nivel de significancia estadística del 5\%. El análisis se desarrolló con el programa de Stata ${ }^{\oplus} 14.0$ y R Statistic para un estudio adicional del post- test en la variable aprendizaje, traducida en rendimiento.

\section{Resultados}

Se aplicó el Inventario de Estilos de Aprendizaje (ILS) de Felder y Silverman a la totalidad del grupo estudiado, encontrándose la mayor prevalencia en el estilo visual, seguido por los sensitivos y secuenciales (tabla 1 ).

\section{Análisis del uso de la estrategia pedagógica con relación al aprendizaje}

Para el análisis cuantitativo correspondiente a la evaluación de las hipótesis enunciadas en 
los apartados anteriores se realizaron pruebas de inferencia estadística, para poder dar respuesta al planteamiento del estudio, donde una estrategia pedagógica basada en soluciones problémicas y estilos de aprendizaje mediada por las TIC mejora el aprendizaje en mecánica respiratoria.

En primera instancia, se propuso que la instrucción de los estudiantes usando materiales adaptados a su estilo de aprendizaje y valiéndose del uso de TIC para administrarlos, no tendría un efecto estadísticamente significativo en el dominio del conocimiento frente a los estudiantes que tomarían la clase tradicional. Esto se midió comparando para cada estudiante su desempeño en el test de selección de única respuesta con opción múltiple administrado al inicio del módulo, y otro de características similares realizado al final del mismo, esta medición apunta a valorar el conocimiento que el estudiante usaría para responder a las preguntas suministradas sobre la mecánica respiratoria.
Se tomaron los datos del pos-test y del pretest para obtener el valor de la variable independiente. En la tabla 2 se presentan los datos de la variación media entre la prueba previa de conocimientos (presaberes) y el resultado en la prueba final de evaluación escrita al final del módulo del curso. Los estudiantes del grupo de intervención presentan una mayor variación promedio en el resultado de la prueba post respecto a la prueba pre $(\bar{x}=0.45, \mathrm{DE}=1.43$, IC $95 \%=-0.007$ - 0.9); mientras en el grupo de control se halló una menor variación entre pruebas, pero no hay mejoría sistemática en el resultado final ( $\bar{x}=.025$, IC $95 \%=-0.49-0.44$ ); es evidente que el valor del intervalo de confianza para el grupo de intervención y de control pasa por el valor de cero con una discreta tendencia a mejor el desenlace para el grupo de intervención, dado que el grado de desmejora fue menor y el de mejora en la calificación fue mayor, frente a esto se considera que el hallazgo podría deberse a la necesidad de un mayor número de muestra que permita decantar el verdadero comportamiento de este hallazgo.

Tabla 1. Estilos de aprendizaje en el grupo estudiado acorde al Inventario de Estilos de Aprendizaje (ILS) de Felder y Silverman

\begin{tabular}{lccc}
\hline \multicolumn{1}{c}{ Estilo de aprendizaje predominante } & Todo el grupo $(\mathbf{n}=\mathbf{8 0})$ & Grupo Intervención $(\mathbf{n}=\mathbf{4 0})$ & Grupo Control $(\mathbf{n}=\mathbf{4 0})$ \\
\hline visual & 26 & 14 & 12 \\
sensitivo & 14 & 8 & 6 \\
secuencial & 14 & 7 & 7 \\
activo & 8 & 2 & 6 \\
reflexivo & 6 & 2 & 4 \\
verbal & 5 & 2 & 3 \\
global & 5 & 3 & 2 \\
intuitivo & 2 & 2 & 0 \\
\hline
\end{tabular}

Fuente: Los autores.

Tabla 2. Comparación de la diferencia entre la calificación de la prueba de presaberes y la prueba escrita final del módulo

\begin{tabular}{ccccc}
\hline Grupo & Diferencia $\bar{x}$ pre - post & DE & IC 95\% & Rango (mínimo - máximo) \\
\hline Control & .025 & 1.458617 & -.4914884 .4414884 & -33 \\
Intervención & .45 & 1.431334 & -.0077629 .9077629 & -33 \\
\hline
\end{tabular}




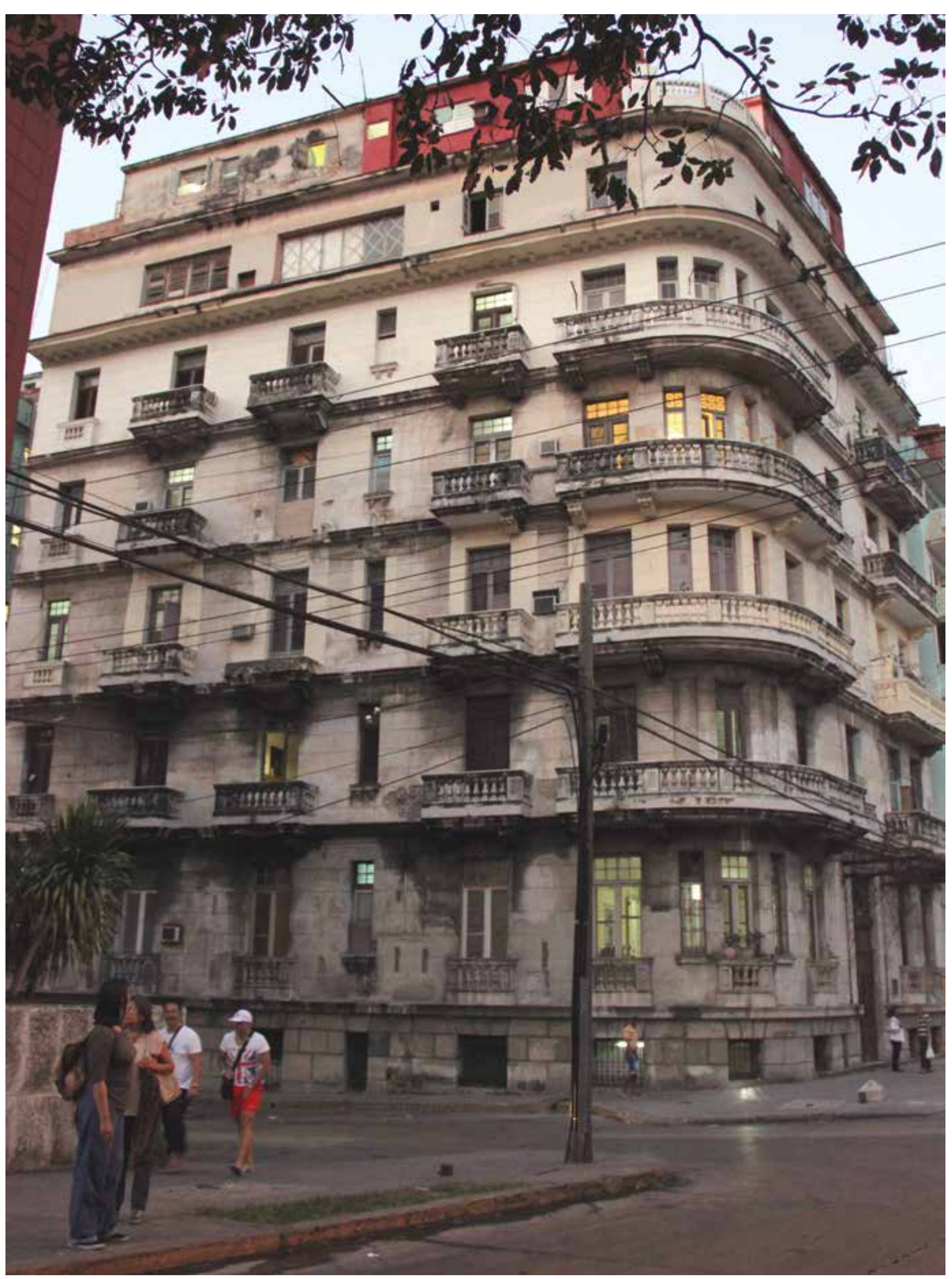


Para evaluar la hipótesis acerca de la existencia de una diferencia estadísticamente significativa en el conocimiento entre el grupo que tomó instrucción usando la estrategia respecto al grupo de control, se realizó una prueba t de Student que arrojó como resultado una diferencia no significativa ( $t=1.4700$, grados de libertad $=78, p=0.145$ ) impidiendo aceptar la hipótesis alterna, en dicho caso se considera que la estrategia pedagógica suministrada al grupo de intervención no mejoró el conocimiento de la mecánica respiratoria en los estudiantes, comparándoles con el grupo de control.

Adicionalmente, con el propósito de valorar si hubo algún cambio entre los mismos participantes y ver si la estrategia pedagógica potenció en algún posible escenario el desempeño en la prueba, se hizo comparación entre la calificación pre-test y pos-test (figura 1) donde se observa una mejoría en la calificación en el grupo de intervención (24 versus 13) comparado con el grupo control para aquellos participantes que aprobaron la prueba; en contraparte, quienes tuvieron un cambio hacia una menor calificación y perdieron fueron ligeramente más numerosos en el grupo de control (13 versus 8 ) frente al grupo de intervención.

Figura 1. Gráfico comparativo en el desenlace de la evaluación escrita pre y pos-test según el grupo (método tradicional versus intervención)

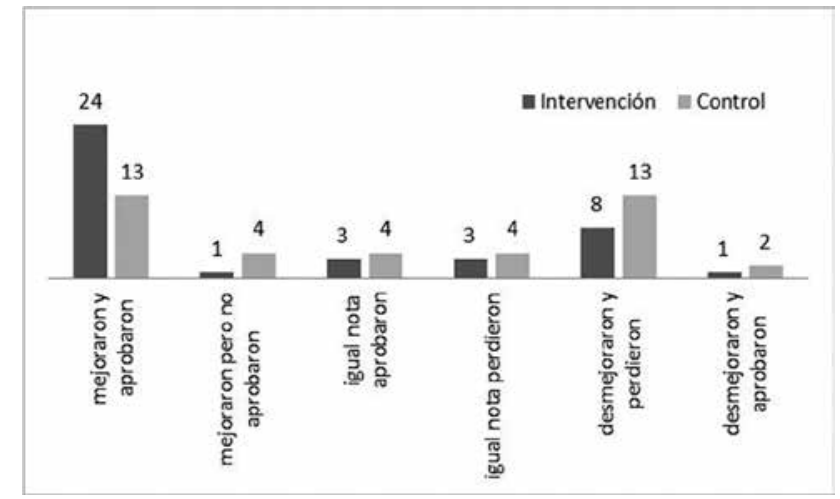

Fuente: Los autores.

Para estudiar las causas que pudieron haber incidido en los resultados previamente descri- tos de la prueba de conocimiento, se realizó un análisis Rasch. Este análisis "predice la probabilidad de respuesta correcta a un ítem en función de la diferencia entre la habilidad de la persona y la dificultad el ítem" (Morgado \& Neusa, 2011, p. 30), por lo tanto si la habilidad supera la dificultad del ítem, la probabilidad de respuesta correcta será mayor, y si la habilidad es menor a la dificultad del ítem, la probabilidad de responder de forma correcta será menor.

Dentro del modelo de Rasch existe una información importante de evaluar y es el ajuste de los ítems al modelo, esto se da porque muchas respuestas pudieron ser al azar, sesgos, o un enunciado en la pregunta no claro. Las medidas de ajuste son dos, el Outfit que es la media de los comportamientos inesperados del participante sobre los ítems alejados de su nivel de habilidad, y el Infit que mide el comportamiento inesperado del participante en los ítems cercano a su habilidad.

Después de hallar el alfa de Cronbach $(a=$ 0.30) se procedió a hallar el índice de dificultad del ítem, usando el programa SPSS, este permite conocer la facilidad o la dificultad al momento de responder, se interpretó como el número de personas que respondieron correctamente un ítem, teniendo en cuenta los siguientes datos: de 0 a 0,15 es un ítem muy difícil, 0,15 a 0,4 difícil, 0,4 a 0,6 es moderado, 0,6 a 0,85 es fácil y de 0,85 a 1.0 es muy fácil (tabla 3 ).

Tabla 3. Resultados e interpretación del índice de dificultad de los ítems

\begin{tabular}{cccc}
\hline Ítem & Media & $\begin{array}{c}\text { Desviación } \\
\text { Estándar }\end{array}$ & Interpretación \\
\hline Ítem1 & .35 & .480 & Difícil \\
Ítem2 & .58 & .497 & Moderado \\
Ítem3 & .64 & .484 & Fácil \\
Ítem4 & .43 & .497 & Moderado \\
Ítem5 & .69 & .466 & Fácil \\
\hline
\end{tabular}

Fuente: Los autores.

Teniendo la dificultad de los ítems se realiza una comparación entre la dificultad de las pre- 
guntas con la habilidad requerida para contestarla usando el análisis Rasch, siendo el punto de corte el $50 \%$ de las probabilidades de resolverla (eje de ordenadas) con su intersecto en la curva de tipo sigmoidea; esta es una forma rápida de evaluar el grado de inteligencia de quien es valorado en una prueba, pues establece una medida arbitraria para comparar al participante con relación a los demás en función de su capacidad para resolver una tarea o pregunta (figura 2). Evidentemente, hay situaciones excepcionales donde una persona podría acertar por azar, o porque iba a seleccionar una respuesta y por error seleccionó otra, entre otros posibles escenarios y es allí donde cobra importancia los ajustes al modelo.

Acorde con los trazados de la gráfica en la figura 2 sobre la dificultad de las preguntas administradas, se corrobora que las preguntas 3 y 5 fueron las de menor dificultad, pues el área bajo la curva es mayor; por el contrario, la pregunta 1 fue la más difícil porque para resolverla el $50 \%$ de oportunidades que se enfrentara se debía tener casi el doble de habilidad que el promedio de los participantes.

Se revisó la variable ítem respecto al ajuste con el modelo Rasch y la calidad del ítem, teniendo como referencia los parámetros definidos por Wring y Linacre, valor que no debe sobrepasar de 0.6 y 1.4 (Rodríguez, 2014) (tabla 4). Con los valores obtenidos se puede concluir que los ítems dentro del test se encuentran ajustados al modelo; los valores de Infit y Outfit concluyen que existe un buen ajuste al modelo, ya que ninguno supera el valor de 1,4.

Tabla 4. Ajuste de los ítems

\begin{tabular}{cccc}
\hline Ítems & P-value* $^{*}$ & Infit MNSQ & Outfit MNSQ \\
\hline Ítem1 & 0.136 & 1.154 & 1.168 \\
Ítem2 & 0.504 & 1.001 & 0.976 \\
Ítem3 & 0.911 & 0.827 & 0.774 \\
Ítem4 & 0.472 & 0.958 & 0.989 \\
Ítem5 & 0.732 & 0.876 & 0.880 \\
\hline
\end{tabular}

* Ios valores deben encontrarse entre +0.05 a 0.1 para que se ajusten al modelo.

Figura 2. Gráfico de probabilidad de respuesta del ítem comparando la habilidad del participante versus la dificultad
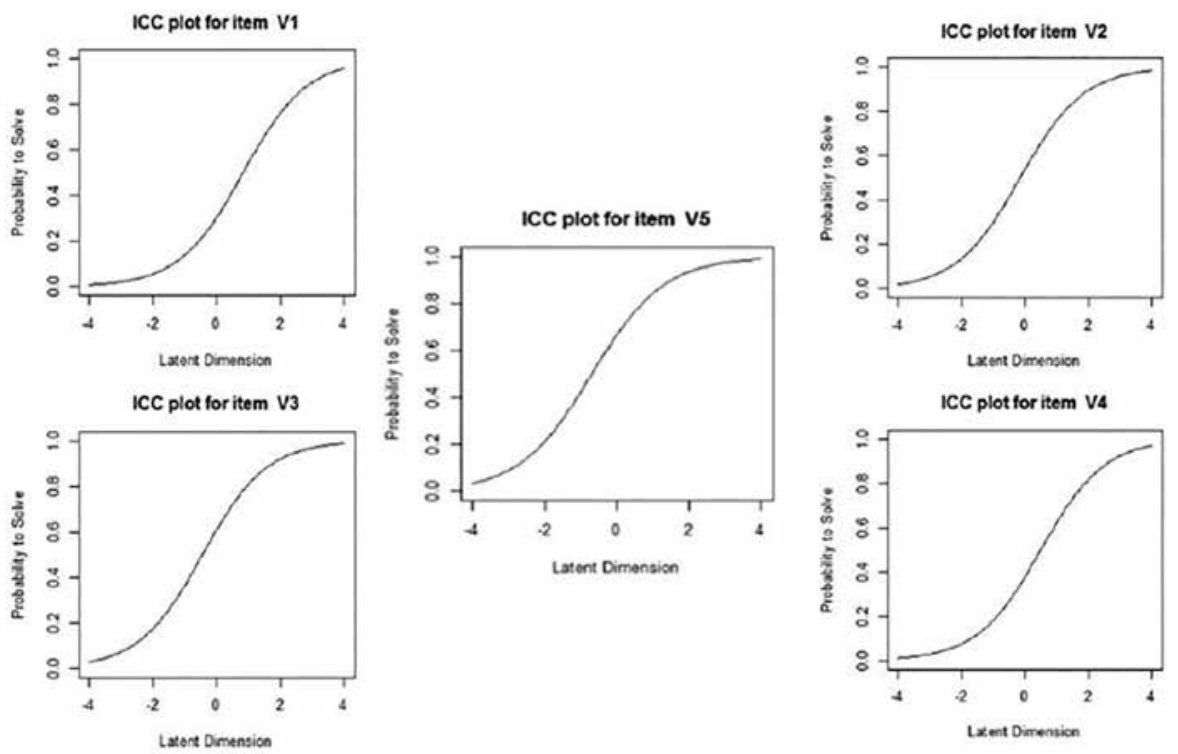

Fuente: Los autores. 
La segunda hipótesis para comprobar enunciaba que el uso de la estrategia pedagógica no mejora el aprendizaje del estudiante para la resolución de problemas de la mecánica respiratoria. Con el propósito de evaluar dicha hipótesis sobre el aprendizaje que permitiese tener mejores competencias en la resolución de situaciones problema empleadas en la actividad de $A B P$, se realizó una prueba $t$ de Student $(t$ $=5.671$ con 78 grados de libertad, $p<0.001$ ) comparando los grupos de intervención ( $\bar{x}=$ 4.05, $\mathrm{DE}=0.268$, IC $95 \%=3.96-4.13)$ y control $(\bar{x}=3.71, \mathrm{DE}=0.263$, IC $95 \%=3.62-3.79)$, respectivamente. Los resultados nos indican que los grupos que tomaron la estrategia basada en situaciones problémicas y de materiales con adaptación al estilo de aprendizaje, mediada por las TIC mejoraron el puntaje obtenido en dicha actividad en comparación con el grupo de control.

Finalmente, se tuvo como tercera hipótesis de trabajo que el uso de la estrategia mediada por situación problémica y TIC no mejora el rendimiento global del estudiante en mecánica respiratoria teniendo en cuenta que la calificación final del módulo tomaba en cuenta la participación en una actividad de grupos (ABP, ponderación $=35 \%$ ) e individual (cuestionario, ponderación $=65 \%)$. Se realizó una prueba t de Student $(\mathrm{t}=2.4296$, grados de libertad $=78, \mathrm{p}$ $=0.017$ ) en la cual se evidenció un mejor rendimiento global en el grupo de intervención ( $\bar{x}$ $=3.33, \mathrm{DE}=0.926$, IC 95\% = 3.03 - 3.62) comparado con el control ( $\bar{x}=2.88, \mathrm{DE}=0.695, \mathrm{IC} 95 \%$ $=2.66-3.10)$.

Se realizaron análisis adicionales para evaluar la existencia de diferencias estadísticamente significativas en el desempeño de los estudiantes en su calificación final asociado a otros factores, siendo significativa en la asociación con el sexo $(p=0.048)$, donde los hombres $(\bar{x}$ $=3.36$, DE $=0.744$, IC95\% = 3.07 - 3.64) obtuvieron notas más altas en comparación con las mujeres $(\bar{x}=2.97, \mathrm{DE}=0.869, \mathrm{IC} 95 \%=2.72$ 3.21); por el contrario, no hubo una asociación de mejor desempeño en la calificación final con respecto al estilo de aprendizaje (ANOVA de una cola, $F=0.52, p=0.81$ ).

\section{Discusión}

Cada estudiante que llega a la universidad trae consigo unos presaberes, habilidades, aptitudes y actitudes diferentes, algunos de estos presaberes no son recientes ni están bien afianzados. En el curso de Fisiología del currículo de Medicina, uno de los contenidos donde los estudiantes muestran más falencias es en el aprendizaje del sistema respiratorio, porque no logran comprender satisfactoriamente los procesos complejos que se producen durante la respiración, debido en gran parte a que no poseen conocimientos de ciertos elementos de la física, requeridos para comprender de forma más fácil el mecanismo del transporte gaseoso que se da en el interior de los alvéolos, así como el movimiento de gases desde la atmósfera hacia el pulmón (Viera, Medina, Díaz, Paz \& González, 2004).

Al igual que ocurre con los presaberes, se observan estudiantes con diferentes estilos de aprendizaje, motivaciones diversas hacia los contenidos de la carrera, ritmos variados de estudio, y velocidades de asimilación de conceptos y destrezas también diferentes, que sumado a otro problema que atañe a la educación médica, como el incremento de contenidos para un desarrollo en el mismo tiempo del semestre, ha hecho visible la necesidad de aprovechar al máximo el tiempo con el estudiante, donde el docente proponga situaciones problemáticas $y$ actividades para que el estudiante realice un aprendizaje activo y colaborativo (Scolari, 2012).

Un estudio realizado por (Ortiz \& Aguilera, 2005) sobre los estilos de aprendizaje de los estudiantes universitarios, menciona la importancia de incorporar estrategias pedagógicas adaptadas al estudiante, ya que al caracterizarlas hacia sus necesidades, favorecen el aprendi- 


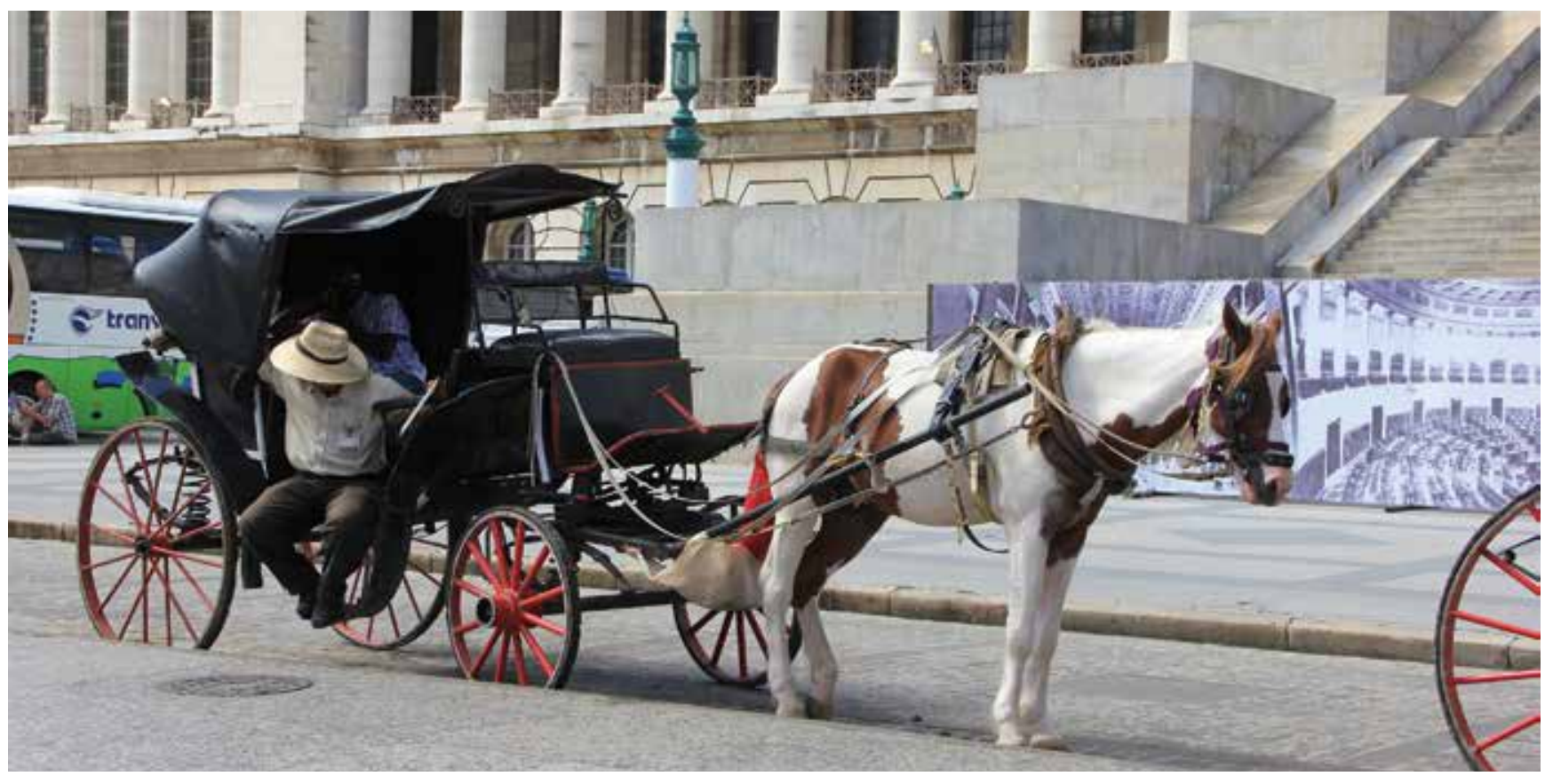

zaje, logrando así obtener una visión más cercana del desempeño profesional.

Este estudio se desarrolló en tres fases: la primera, el diseño de la estrategia; la segunda, la implementación y, tercera, la evaluación. En el diseño del micro currículo se tuvo en cuenta el modelo TPACK con el fin de integrar los tres conocimientos planteados: conceptos que incluye revisión del currículo, requerimientos de dominio, los contenidos propios de la materia de Fisiología y las patologías respiratorias que mayor error de diagnóstico presentan; pedagogía que comprende la didáctica, los requerimientos psicopedagógicos que pertenecen al enfoque teórico y práctico de la educación, tales como el aprendizaje basado en situaciones problémicas, los estilos de aprendizaje de cada uno de los estudiantes, el nivel de conocimientos que tiene el estudiante y sus factores físicos, y la tecnología que se tendrá en cuenta para los requerimientos necesarios en la interfaz donde se utilizará la plataforma Moodle, para la incorporación de los instrumentos y herramientas adaptados a las necesidades propias de cada estudiante (Herrera, 2004).

El marco muestral fue la totalidad de los estudiantes de medicina de una universidad co- lombiana que cursan tercer semestre, son aproximadamente ochenta alumnos por cada cohorte; esto pone de presente que la población seleccionada para realizar esta investigación educativa es bastante cercana a la usual dentro de las diferentes cohortes de dicha universidad que toman el curso de Fisiología.

El test psicométrico es una técnica derivada de la entrevista y de la encuesta que tiene como objeto lograr información sobre rasgos definidos de la personalidad, la conducta o determinados comportamientos y características individuales o colectivas de la persona. Esta es evaluada y observada por el investigador y se aplica a través de preguntas o actividades (Huaman, 2005). A este grupo le fue aplicado al inicio del semestre el cuestionario de Felder y Silverman con el fin de identificar los estilos de aprendizaje más frecuentes, es importante anotar que sería adecuado que se correlacione posteriormente con el uso de la herramienta que haga cada uno de estos estudiantes, porque puede suceder que los resultados del cuestionario no sean del tipo de materiales usados predominantemente por el sujeto investigado. Los resultados de dicha batería encontraron como estilo más predominante el visual (26 participantes), seguido por el sensitivo y se- 
cuencial (14 participantes cada uno) y el menos frecuente fue el intuitivo (2 participantes); cabe anotar que esto hace referencia al estilo más predominante y que el constructo de los autores de dicha batería psicométrica es que existen 4 dominios de estilos de aprendizaje y el estado óptimo sería hallarse en un punto neutral para los 4 dominios, de forma que la persona se sentiría cómoda con cualquier estrategia pedagógica en su proceso de enseñanza-aprendizaje. Es posible que el alto número de personas con estilo predominantemente visual sea consecuencia del modelo tradicional de educación escolarizada y que se prolonga en los escenarios universitarios.

El test de conocimientos fue usado en dos ocasiones: pre-test (prueba de presaberes) y pos-test (al finalizar el módulo); es decir, se pretendió conocer en primera instancia cómo interactuaba el estudiante con las herramientas desarrolladas para su aprendizaje; luego de aplicada la estrategia pedagógica que los investigadores diseñaron, se reiteró la aplicación del test para conocer si la herramienta pudo ser efectiva en la aplicación con los estudiantes.

En el presente estudio el desempeño fue ligeramente diferente entre hombres y mujeres, en contraste con los hallazgos de García, Arras, González, Hernández, Martín, Prada... y Torres (2010), donde no hay excesivas diferencias entre géneros, aunque los hombres consiguen puntajes más altos en comparación con las mujeres; es posible que estos resultados obtenidos se relacionen con el hecho de que los grupos de estudiantes universitarios en este programa de Ciencias de la Salud sean mayoritariamente mujeres.

En cuanto a la existencia de relación entre rendimiento académico y los estilos de aprendizaje a pesar de no ser significativa la relación, demuestra que cada individuo aprende de manera diferente, tal como lo afirman (Fontalvo et al., 2007) los estilos de aprendizaje tienen buen nivel de aceptación y satisfacción de parte de los estudiantes, por esta razón se hace importante que los contenidos estén cada vez más estrictamente adaptados al estilo de aprendizaje que ellos presentan. Se hizo una exploración de enfoque mixto sobre la satisfacción con los materiales adaptados a los estilos de aprendizaje, en la cual los estudiantes participantes en el grupo de intervención evaluaron aspectos relacionados con el desarrollo del curso (satisfacción general = $79.26 \%$ ), lo que es indicativo de que hubo una buena aceptación por parte de los estudiantes, teniendo en cuenta que se trata de una metodología a la cual no se hallan acostumbrados y que se espera que haya un cierto grado de resistencia en los estudiantes al cambiárseles el escenario usual de su proceso de enseñanza-aprendizaje.

Al valorar si hubo diferencia en el desempeño entre las pruebas de conocimiento de presaberes y la del final del módulo, no se halló diferencia estadísticamente significativa entre ambos grupos $(p=0.14)$. Esto puede deberse a que las preguntas evaluarón únicamente la recuperación de información (memorización) o circunscribirse a aspectos del estado en que los estudiantes enfrentaron la prueba de evaluación de conocimiento, ya que la instrucción fue en espacios y tiempos diferentes, pero no era posible controlar las acciones del tiempo de trabajo independiente de los participantes, por ende, es posible que hubiesen compartido informaciones entre ellos o se hubieran reunido a preparar la evaluación en grupos de estudio donde participaban los de metodología tradicional, mezclados con el grupo de intervención, y de esta forma se pudo haber diluido alguna diferencia no atribuible al azar, dado el valor de p en la prueba t. Por esta razón se decidió analizar a través del método de Rasch las preguntas formuladas en función de su probabilidad de responderla y su dificultad como un método para evaluar el desempeño de los estudiantes dentro de esas dimensiones implícitas en la tarea de responder a un cuestionario. 
Antes de realizar las medidas estadísticas para el análisis Rasch, se decidió encontrar primero el alfa de Cronbach y el índice de dificultad el ítem. Se halló el alfa de Cronbach a través del software SPSS, obteniendo como resultado un valor de .30, con una confiabilidad media. Cabe resaltar que según Campo y Oviedo (2008), la prueba de Kuder-Richardson (KR-20) y el coeficiente del alfa de Cronbach, solo se pueden calcular apropiadamente en escalas para medir características o atributos, no se recomienda para pruebas de conocimientos, debido a que necesitan tener conocimiento previo sobre el tema. Por esta razón se decidió realizar un análisis más profundo respecto al instrumento para evaluar el rendimiento académico en el estudiante.

Es notorio el resultado de la actividad de resolución de problemas, pues en ella el desempeño del grupo de intervención (calificación promedio $=4.05$ sobre 5 ) fue superior al grupo de control (calificación promedio $=3.71$ sobre 5) hallándose diferencia significativa ( $\mathrm{t}=5.671$ con 78 grados de libertad, $p<0.001$ ); es posible que estos resultados se deban a que es una actividad de carácter grupal y al haberse encontrado un mejor desempeño en los grupos que recibieron la instrucción mediada por TIC, vale la pena tener en consideración estos hallazgos para hacer un estudio más detallado del mismo y poder descubrir los factores que podrían estar desencadenando un mejor resultado, algunos de ellos posiblemente sean relacionados con la dinámica de trabajo en grupo, como habilidades de organización, toma de decisiones, negociación o también los relacionados con factores individuales, como una menor sensación de presión por el resultado, sentirse respaldado, entre otros.

También vale la pena tener en cuenta el resultado global de la calificación promedio sobre cinco puntos posibles que se logró al terminar el módulo y que incluyó los resultados individuales y grupales, evidenciándose un mejor desempeño en el grupo de intervención que en el de control (3.33 vs 2.88 , respectivamente, $p=0.017$ ); esto invita a que se realicen procesos evaluativos más amplios, diversos e incluyentes, puesto que permiten una evaluación más completa del estudiante en cuanto a sus diferentes dimensiones y de seguro más orientada al logro de las competencias que requiere el futuro profesional.

\section{Conclusiones}

La incorporación de TIC a la enseñanza en las escuelas de Medicina ha sido algo lenta y posiblemente esto se deba a prevención y reticencia de parte de los docentes que conducen estas cátedras; en el presente trabajo de investigación se pudo evidenciar que en cuanto al desempeño individual, la estrategia pedagógica mediada por TIC al menos genera los mismos resultados que el curso presencial tradicional y como valor agregado parece mejorar aspectos cognitivos relacionados con la resolución de problemas.

Es una misión importante el desarrollo de iniciativas de investigación de tipo multicéntrico que permitan tener un conocimiento más amplio sobre las consecuencias de los cambios que se aplican a los diferentes programas educativos, así como determinar si los procesos educativos innovadores son susceptibles de ser aplicados en lugares culturalmente diferentes y con las mismas posibilidades de éxito. Asimismo, se abre la puerta al intercambio y enriquecimiento de la trayectoria académica, la mejora en la calidad de la pedagogía, el arbitraje y discusión académica entre docentes, todo esto en conjunto muy probablemente permitirá el desarrollo cambios en beneficio de los estudiantes.

El apoyo de plataformas de administración del conocimiento (LMS) permite generar nuevos espacios de interacción entre docentes y estudiantes, en esto es importante la adaptación del entorno educativo para hacerlo amigable para docentes y estudiantes al máximo posible; 
también cobran notoria importancia los aspectos técnicos de las LMS, pues ante los fallos, generalmente los usuarios pierden la expectativa sobre estas, perdiéndose así la oportunidad de revolucionar y complementar la educación.

La determinación de los estilos de aprendizaje de los estudiantes al iniciar los periodos académicos es una tarea que permite adaptar y reorientar algunas actividades de los cursos de educación, a partir de esto es posible facilitar y allanar el camino para que los estudiantes puedan lograr las competencias requeridas en un ambiente de enseñanza-aprendizaje más constructivo y que verdaderamente favorezca el logro de los objetivos propuestos en la noble labor de los docentes, convirtiéndolos en mediadores de un proceso activo que a largo plazo podrá dejar una huella más significativa en los estudiantes.

\section{Referencias}

Briones, G. (1996). Metodología de la investigación cuantitativa en las ciencias sociales. Bogotá, D.C., Colombia: Instituto Colombiano para el Fomento de la Educación Superior ICFES.

Byrne, N., \& Rozental, M. (1994). Tendencias actuales de la educación médica y propuesta de orientación para la educación médica en América Latina. Educ Med Salud, 28(1), 53-93.

Campo, A., \& Oviedo, H. (2008). Propiedades psicométricas de una escala: la consistencia interna. Revista de Salud Pública, 10(5), 831-839. http://doi.org/10.1590/S012400642008000500015

Crispin, M. I., Doria, M. C., Rivera, A. B., De la Garza, M. T., Carrillo, S., Guerrero, L., Athié, M. J. (2011). ¿Qué es el aprendizaje y cómo aprendemos? En Aprendizaje Autónomo. Orientaciones para la docencia ( ${ }^{\text {a }}$ ed., pp. 10-28). Mexico: Universidad Iberoamericana.

Díaz, M. (2006). Modalidades y métodos de enseñanza centrados en el desarrollo de competencias. Barcelona. Recuperado de http://www.publicacions.ub.edu/revistes/ edusfarm0/news/mario_de_miguel.pdf

Duque F, G. A., \& Perdomo Q, M. D. (2014). Conceptos de evaluación en relación con los paradigmas psicológicos de la educación. Revista Amazonia Investiga, 3(5), 103-118.

Felder, R. M., \& Spurlin, J. (2005). Applications, Reliability and Validity of the Index of Learning Styles. International Journal of Engineering Education, 21(1), 103-112. http:// doi.org/0949/-149X/91

Felder, R., \& Soloman, B. (2005). Index of Learning Styles (ILS). Retrieved April 13, 2016, from http://www4.ncsu.edu/ unity/lockers/users/f/felder/public/ILSpage.html
Fontalvo, H., Iriarte, F., Domínguez, E., Ricardo, C., Ballesteros, B., Muñoz, V., \& Campo, J. (2007). Diseño de ambientes virtuales de enseñanza-aprendizaje y sistemas hipermedia adaptativos basados en modelos de estilos de aprendizaje. Zona Próxima, (8), 42-61.

Freire, P. (2004). Pedagogía de la autonomía. (Reseña del libro). Paz e Terra SA, Ed. Brasil: Sao Paulo. Recuperado de http://www.buenosaires.gob.ar/areas/salud/dircap/ mat/matbiblio/freire.pdf

García, A., Arras, A., González, L., Hernández, A., Martin, J., Prada, S., Torres, C. (2010). Competencias en TIC y rendimiento académico en la universidad: diferencias por género. Salamanca, Autónoma de Chihuahua y Veracruzana. Recuperado de http://www.worldcat. org/title/competencias-en-tic-y-rendimiento-academico-en-la-universidad-diferencias-por-genero/ oclc/733969316\&referer=brief_results

Herrera, M. (2004). Las nuevas tecnologías en el aprendizaje constructivo. Iberoamericana de Educación, 34(4), 1-20. Recuperado de http://rieoei.org/tec_edu29.htm

Huaman, H. (2005). Manual de técnicas de investigación: Conceptos y aplicaciones ( $2^{\mathrm{a}}$ ed.). Lima, Peru: IPLADEES, S.A.C. Recuperado de http://trabajodegradobarinas.blogspot. com.co/2014/04/manual-de-tecnicas-de-investigacion. html

Koehler, M., Mishra, P., Akcaoglu, M., \& Rosenberg, J. (2008). The Technological Pedagogical Content Knowledge Framework for Teachers and Teacher Educators (pp. 1-7).

Morgado, C., \& Neusa, M. (2011). Análisis de las olimpiadas regionales de matemáticas UIS implementando el modelo Rasch para los años 2009 y 2010. Universidad Industrial de Santander.

Ortiz, E., \& Aguilera, E. (2005). Los estilos de aprendizaje de los estudiantes universitarios y sus implicaciones didácticas en la educación superior. Pedagogía Universitaria, 10(5).

Rodríguez, M. (2014). Validez y confiabilidad de un instrumento de calidad de vida relacionado con la salud (KIDSCREEN-52) en niños y adolescentes escolarizados de la ciudad de Bucaramanga. Universidad Industrial de Santander.

Scolari, C. (2012). Universidad y crisis (III): La clase magistral. Recuperado el 1 de september de 2015, de http://www. digitalismo.com/2004-2014/universidad-y-crisis-iii-laclase-magistral/

Siemens, G. (2014). Connectivism: A Learning Theory for the Digital Age. Canada. Recuperado de http://er.dut. ac.za/bitstream/handle/123456789/69/Siemens_2005_ Connectivism_A_learning_theory_for_the_digital_age. pdf? sequence $=1$ \&isAllowed $=y$

Vera, F. (2008, mayo). La modalidad blended-learning en la educación superior, 23, 1-16.

Viera, L., Medina, L., Díaz, P., Paz, C., \& González, O. (2004). Sistema tutorial para el estudio de la fisiología del aparato respiratorio del cuerpo humano. Educación Médica Superior, 18(3), 1. Recuperado de http://scielo.sld.cu/scielo.php?script=sci_arttext \&pid=S086421412004000300004\&lng=es\&nrm=iso\&tlng=es 